\title{
Nonlinear Cointegration Analysis Based on Support Vector Machines and Its Application to the Connection between Financial Markets
}

\author{
Shen Chuanhe, Liu Zhongwen, Li Ying \\ Institute of Financial Engineering of Shandong Women's University \\ Jinan, China \\ schuanhe@sina.com, lzw3541@sohu.com, cherry_jn@126.com
}

\begin{abstract}
With the purpose of analyzing non-stationary time series, this paper introduced a novel nonlinear cointegration discriminate analysis by employing support vector machines to overcome existing limitations of two methods, that is, the statistical approach and the neural network used by the nonlinear cointegration theory. The proposed nonlinear cointegration discriminate analysis can effectively address the key step in the nonlinear cointegration test. Then, the application of the innovated method in the investigation of the connection between financial markets was explored. The empirical analysis demonstrates that the support vector machine is significant in investigating the money demand function and its stability and has advantages in dealing with nonlinear cointegration relations among different financial variables and estimating nonlinear function over the two methods above.
\end{abstract}

Keywords-non-stationary time series; nonlinear cointegration analysis; support vector machine; neural network; the connection between financial markets

\section{INTRODUCTION}

For the sake of the nonlinear and no-stationary financial time series, many scholars have developed various econometrics models to tackle effectively relations among different financial variables. As one of the most important methodologies, the nonlinear cointegration theory, an extension of the basic linear cointegration theory initiated by Granger (1981)[1], Engle and Granger (1987) [2], and Johansen $(1988,1991)$ [3-4], has showed powerful capability of depicting the relations. But, discriminate analysis of whether the relations are nonlinear cointegration constitutes the key step in the nonlinear cointegration test. So far, most literatures concern the test, such as Dufrénot and Mignon (2002) [5], Chang and Park (2003) [6], Lieberman and Phillips (2008) [7], Choi and Saikkonen (2010) [8], etc. These methods can be divided into two categories, that is, the parametric approach and the nonparametric one.

Specifically, nonparametric estimates of nonlinear cointegration have been computed from data by Granger and Hallman (1991) [9], and Chang et al (2001) also proposed efficient nonstationary nonlinear least square (EN-NLS) to improve efficiency in NLS estimation [10]. Contrast to this, the nonparametric approach, deeply partitioned into two subcategories, i.e. the nonlinear transform of variables and the intelligence methods, has improved the nonlinear cointegration discriminant analysis. Grange and Hallman
(1991) pioneered the nonlinear transform of variables, socalled nonparametric alternating conditional expectation algorithm, which only demands distribution hypothesis weakly and can thus tackle various nonlinear transform [9] Dufrénot and Mignon (2002) and Zhang et al. (2008) employed neural network technology in succession to cope with parameter estimation of the nonlinear cointegration relations by taking advantage of its good approximation capability of the nonlinear cointegration functions [5][11].

But, all of the two categories above have limitations in nonlinear cointegration test. The parametric approach, attributable to econometrics, contains so many strict prior hypothesis inconsistent with real financial markets that some drawbacks inevitably arise, like too many model parameters and lower test effectiveness under the small-sample condition (Escribano et al, 2006; Esteve and Prats, 2010) [12-13]. While, the neural network has still some problems, such as local optimum, over fitting, and so on (Karlsen et al, 2007a) [14].

Therefore, this paper attempts to propose another test method based on support vector machines, compared with the neural network methodology, to allow for the asymptotic properties of nonparametric estimators either for nonlinear error correction or cointegration models (Karlsen et al, 2007b) [15]. In section II, the methodology is described to explore multi-variables financial time series analysis. Section III presents its application to analysis of the connection between the money market and the capital market, with the conclusion in the final section.

\section{NONLINEAR COINTEGRATION ANALYSIS BASED ON SUPPORT VECTOR MACHINES}

The support vector machine is first introduced underneath, with the nonlinear cointegration analysis innovated based on it.

\section{A. Support Vector Machines}

The support vector machine is a new generation learning system for small samples and is constructed according to the statistical learning theory by Vapnik (1995) [16], which employs the structural risk minimization principle other than the empirical risk minimization principle generally used by the neural network methodology. It therefore overcomes the shortcomings of the neural network recognition algorithm, such as large samples, "the curse of dimensionality", local optimization, over-fitness, etc., and has a better model generalization performance. It is now being established as 
one of the standard tools for machine learning and data mining, such as pattern recognition, regression analysis and probability density estimation [17]. Here, the support vector regression machine ( $\varepsilon$-SVR hereafter), as one kind of support vector machines, is introduced below [18-20].

The $\varepsilon$-SVR is involved in the approximation of function, that is, the selection of a special function in an image space through machine learning, with the function expressed as follows:

Given a training set, $\left\{X_{i}, y_{i}\right\}, i=1,2, \ldots, l$, where the input data $X$ is assumed to be a compact domain in a Euclidean space $R^{n}$ and the output data $y$ is assumed to be a closed subset of $R$. Learning from data can be viewed as an approximation of the multivariate function $f(X)$ which represents the relation between the input $X$ and the output $y$. By some nonlinear mapping $\phi(X)$, the input $X$ is mapped onto a hypothesis space (or feature space) in which the learning machine (algorithm) selects a certain function $f(X)$.

According to the learning theory, for constructing a nonlinear support vector machine, the decision function takes the following form:

$$
f(X)=W \cdot \phi(X)+b
$$

Considering the $\varepsilon$-insensitive cost function:

$$
M(y, f(X))=L\left(|y-f(X)|_{\varepsilon}\right)
$$

where $|y-f(X)|_{\varepsilon}=\left\{\begin{array}{ll}0, & i f|y-f(X)| \leq \varepsilon \quad \\ |y-f(X)|-\varepsilon, & \text { others }\end{array} \quad\right.$ and $\varepsilon$ represents the insensitivity range of the $\varepsilon$-SVR.

This means that the cost is equal to 0 if the deviation of the expected value from the observed value is smaller than the $\varepsilon$.

Then, solving regression problem is equivalent to optimizing the following problem:

$$
\begin{gathered}
\min \frac{1}{2}\|W\|^{2}+C\left(\sum_{i=1}^{l} \xi_{i}^{*}+\sum_{i=1}^{l} \xi_{i}\right) \\
\text { s.t. } y_{i}-\left(W \cdot \varphi\left(X_{i}\right)\right)-b \leq \varepsilon+\xi_{i}^{*}, i=1,2, \cdots, l \\
\left(W \cdot \varphi\left(X_{i}\right)\right)+b-y_{i} \leq \varepsilon+\xi_{i}, i=1,2, \cdots, l \\
\xi_{i}^{*} \geq 0, i=1,2, \cdots, l \\
\xi_{i} \geq 0, i=1,2, \cdots, l
\end{gathered}
$$

where $C$ is the regularization constant which plays a tradingoff between the regularization performance and the empirical error, and $\xi_{i}, \xi_{i}^{*}$ represent slack variables. follows:

The corresponding dual problem can be expressed as

$$
\begin{array}{ll}
\min & \frac{1}{2} \sum_{i=1}^{l}\left(\alpha_{i}^{*}-\alpha_{i}\right)\left(\alpha_{j}^{*}-\alpha_{j}\right) K\left(X_{i}, X_{j}\right) \\
& +\varepsilon \sum_{i=1}^{l}\left(\alpha_{i}^{*}+\alpha_{i}\right)-\sum_{i=1}^{l} y_{i}\left(\alpha_{i}^{*}-\alpha_{i}\right) \\
\text { s.t. } & \sum_{i=1}^{l}\left(\alpha_{i}^{*}-\alpha_{i}\right)=0 \\
& 0 \leq \alpha_{i}^{*}, \alpha_{i} \leq C \quad i=1,2, \cdots, l
\end{array}
$$

By solving this quadratic programming problem above, the $\mathrm{w}$ and $\mathrm{b}$ in (1) can be obtained:

$$
w=\sum_{i=1}^{l}\left(\alpha_{i}^{*}-\alpha_{i}\right) \varphi\left(X_{i}\right)^{\prime} \quad b=y_{j}-\sum_{i=1}^{l}\left(\alpha_{i}^{*}-\alpha_{i}\right) K\left(X_{i}, X_{j}\right)+\varepsilon \cdot
$$

The decision function represented in (1) is then transformed into:

$$
f(X)=\sum_{i=1}^{l}\left(\alpha_{i}^{*}-\alpha_{i}\right) K\left(X_{i}, X\right)+b
$$

\section{B. Nonlinear Cointegration Analysis Based on $\varepsilon-S V R$}

The cointegration theory falls into two classes, that is, linear cointegration theory and nonlinear cointegration one. To the linear cointegration theory, the cointegration reflects station degree variability of various no-stationary stochastic series after linear combination. Engle and Granger (1987) put forward the cointegration notion, which establishes theory base for us to seek equilibrium relations among two or more no-stationary stochastic series and to set up error correction models for the variables with cointegration relations [2]. What is more, it integrates advantages of shortterm and dynamic models used by time series analysis method to determination of the long-term stable relations in econometrics. But, nonlinear cointegration method is being much appreciated because financial time series show the characteristics of strong no-station. Here, only the nonparametric method is discussed and much emphasis will be played on the machine learning approach, such as the support vector machine. The principal idea is that the $\varepsilon$-SVR is underneath used to improve the discriminate analysis, with the definition of nonlinear cointegration formulated fist as follows:

If $X$ is a $m \times 1$ order time series vectors, and satisfies [5][11]:

The component of $\left\{X_{i}\right\},\left\{x_{i j}\right\}$, is a long-memory time series (LMT) or $I(1)$ time series $(i=1,2, \ldots, l ; \mathrm{j}=1,2, \ldots, m)$, and there exists a function $f(\cdot)$, which makes $y_{i}=f\left(x_{i 1}, x_{i 2}, \cdots, x_{i m}\right)$ a zero-mean short memory in mean (SMM) or $I(0)$.

Then, the nonlinear cointegration relation exists among all the component time series of $\left\{X_{i}\right\}$.

Obviously, how to estimate the nonlinear function $f(\cdot)$ becomes the emphasis and the difficulty in nonlinear cointegration analysis, and many methods have been developed for this so far. Zhang et al (1999) employed a neural network to test the existence of nonlinear 
cointegration relation and discussed its feasibility [5]. But, the neural network is still flawed to the discriminate analysis of nonlinear cointegration because of its well-known limitations. Here, the $\varepsilon$-SVR will be applied to overcome these limitations and the specific steps are expressed as follows according to the definition [5][11]:

- Choose financial variable time series as input vectors $X_{i}$ of the $\varepsilon$-SVR, with a known shortmemory time series, $\left\{D_{i}\right\}$, used simultaneously as supervised output (i.e. the output vector $\left\{y_{i}\right\}$ ).

- Employ the $\varepsilon$-SVR to estimate the nonlinear function $y_{i}=f\left(x_{i 1}, x_{i 2}, \cdots, x_{i m}\right)$.

- Obtain the estimated output value $\hat{y}_{i}$ using $\hat{y}_{i}=f\left(x_{i 1}, x_{i 2}, \cdots, x_{i m}\right)$ with the $X_{i}$ still the input vector of the $\varepsilon$-SVR.

- Test existence of the nonlinear cointegration relation among component vectors of $\left\{X_{i}\right\}$. This means verifying the long memory characteristic in the time series $\left\{\hat{y}_{i}\right\}$.

If there is still the long memory characteristic in the time series $\left\{\hat{y}_{i}\right\}$, the nonlinear cointegration relation among component vectors of $\left\{X_{i}\right\}$ doesn't exist. Otherwise, this kind of relation is existent.

\section{APPLICATION TO ANALYSIS OF THE CONNECTION BETWEEN FINANCIAL MARKETS}

Actually, the nonlinear cointegration analysis is widely used in financial issues, such as asset pricing (Lieberman and Phillips, 2008; Amano, et al, 2012) [7][21]. Here, the nonlinear cointegration analysis based on SVM will be applied in the investigation of the connection between financial markets.

With the rapid development of the capital market, its impact on the macroeconomy, the money market and till the monetary policy is emerging and becoming more significant. It is the movements in stock prices that influence these respects primarily through two channels, aggregate consumption and household wealth [22]. Because of their potential impact, stock price movements are likely to be an important determinant of monetary policy decisions, and many quantitative analysis methods have consequently been developed to deal with the connection between the money market and the capital market.

As a prevailing method in this field, the cointegration theory is increasingly innovated and the approach based on the $\varepsilon$-SVR above contributes to the innovation. Here, the approach is employed to analyze the connection and the emphasis will aim to long-term equilibrium relation between the two markets. The main task below is to explore the influence from the capital market on the money demand function and its stability, concerning the changes in influencing factors of the function.

\section{A. The Money Demand Function and Its Stability}

The money demand function mainly refers to the direction and extent of affection, from the changes in quantity of money, on economic process, and its stability is still an existing important problem [23]. The influencing factors of the function can be separated into three categories: the first is scale variables, including wealth and income; the second is opportunity cost variables, like the yield of the asset except for money and the third is other variables, such as asset price risk, monetization of economy, financial institutional reform, relations among the internal states of the financial system, etc.

Here, the money demand function can be denoted as follows according to [24]:

$$
\Delta M_{2}=f\left(r_{3-m}, r_{30-y}, r_{\text {bank }}, \Delta_{\text {income }}, \Delta_{\text {index }}^{+}, \Delta_{\text {index }}^{-}\right)
$$

In the meantime, in order to deeply investigate the changes in demand quantity of money caused by the changes in stock index, the $\Delta_{\text {index }}$ is subdivided into two new factors, that is, $\Delta^{+}{ }_{\text {index }}$ and $\Delta_{\text {index }}^{-}$[25]. The rules for their values are as follows: if the $\Delta_{\text {index }}$ is positive, the $\Delta^{+}$index is just equal to the $\Delta_{\text {index }}$; otherwise, it takes 0 . The formulation is the same for the $\Delta_{\text {index }}^{-}$, which means the $\Delta_{\text {index }}^{-}$is just set the same value as the $\Delta_{\text {index }}$ if the $\Delta_{\text {index }}$ is negative; otherwise, it takes 0 .

In fact, the stability of the function, that is, the stability of the relations among variables, is more important than the variables themselves because not only the variables but also their relations vary with economic system, economic structure, financial instruments and financial innovation, etc. All these result in the changes in the function and affect monetary policy efficiency, which deeply reflects the complexity of the function. Therefore, it is necessary that some sophisticated measures should be taken to analyze the complexity, and the nonlinear cointegration method based on the $\varepsilon$-SVR is just for this.

\section{B. Empirical Analysis}

Here, the $\triangle M_{2}$ is chosen as the output of the $\varepsilon$-SVR, $y_{i}$, and six influencing factors constitute the input vector, $X_{i}$, including $r_{3-m}, r_{30-y}, r_{\text {bank }}, \Delta_{\text {income }}, \Delta_{\text {index }}^{+}$and $\Delta_{\text {index }}^{-}$. Therefore, the input vector, $X_{i}$, hence becomes six-dimensional, i.e. $X_{i} \in R^{6}$.

The monthly data for $X_{i}$ and $y_{i}$ is collected from $31 / 8 / 2003$ to $31 / 8 / 2010$, with 84 input vectors and output values obtained respectively, which means $l=84$ in (2).

The radial basis function is chosen as the kernel function of the $\varepsilon$-SVR in (2), with the expression as follows:

$$
K\left(X_{i}, X_{j}\right)=\exp \left(-\left\|X_{i}-X_{j}\right\|^{2} / \sigma^{2}\right)
$$

where $\sigma^{2}$ is the width of the radial basis function.

After the parameters $C$ and sig2 $\left(\sigma^{2}\right)$ are set 10 and 0.2 respectively, the demand function of money in (3) comes arise after the $\varepsilon$-SVR in (2) is trained by all of $X_{i}$ and $y_{i}(i=1$, $2 . ., 84)$ using MATLAB software.

In order to analyze the sensitivity of the demand function of money to every influencing factor, take underneath the 
partial derivative of the function, that is the output $f\left(x_{i}\right)$, with respect to the input $x_{i k}$ according to (2) [26]:

$$
\begin{aligned}
& \frac{\partial y_{i}}{\partial x_{i k}}=\frac{\partial\left(\sum_{i=1}^{l}\left(\alpha_{i}^{*}-\alpha_{i}\right) K\left(X_{i}, X_{j}\right)+b\right)}{\partial x_{i k}} \\
& =-\frac{2}{\sigma^{2}} \sum_{j=1}^{l_{s}}\left(\alpha_{j}^{*}-\alpha_{j}\right)\left(x_{i k}-x_{j k}\right) \exp \left(-\frac{1}{\sigma^{2}} \sum_{l=1}^{n}\left(x_{i l}-x_{j l}\right)^{2}\right)
\end{aligned}
$$

where $x_{i k}(i=1,2, \ldots, 84 ; k=1,2, \ldots, 6)$ represents the $K^{\text {th }}$ component of the sample input vector, $X_{i}$, and $l_{s}$ is the number of support vectors.

All the values of the sensitivity of the function can be obtained by (4) and shown in Table I, with the symbol "+" standing for the number of positive sensitivity values, "-” the number of negative sensitivity values and " $A A V$ " the averaged absolute value of all the sensitivity values. Therefore, the $A A V$ of the $K^{\text {th }}$ component of $X_{i}$ can be expressed as follows:

$$
A A V=\frac{1}{l}\left|\frac{\partial y_{i}}{\partial x_{i k}}\right| .
$$

Then, the structure of interaction between the money demand function and every influencing factor, reflecting short-term changes in their direction and extent to which the function varies correspondingly, can be found through the analysis of $A A V$ in (5) and the results are all shown in Table I.

TABLE I. THE SENSITIVITY OF MONEY DEMAND FUNCTION TO INFLUENCE FACTORS

\begin{tabular}{|c|c|c|c|c|c|c|}
\hline & $\boldsymbol{r}_{3-\mathrm{m}}$ & $\boldsymbol{r}_{\text {30-y }}$ & $\boldsymbol{r}_{\text {bank }}$ & $\Delta_{\text {income }}$ & $\Delta_{\text {index }}^{+}$ & $\Delta_{\text {index }}^{-}$ \\
\hline+ & 4 & 7 & 5 & 78 & 72 & 13 \\
\hline- & 80 & 77 & 79 & 6 & 12 & 71 \\
\hline Total & 84 & 84 & 84 & 84 & 84 & 84 \\
\hline$A A V$ & 0.16 & 0.31 & 0.27 & 0.24 & 0.51 & 0.42 \\
\hline
\end{tabular}

Obviously, it is shown in Table I that in all influencing factors, only $\Delta^{+}$index and $\Delta^{-}$index display instable interaction with the demand quantity of money.

Now, the nonlinear cointegration analysis based on the $\varepsilon$ SVR is implemented underneath in order to deeply explore whether the long-term stable and balanced relation between the demand quantity of money and the $\Delta_{\text {index }}$ is existent. But, the input vector of the $\varepsilon$-SVR needs to be adjusted before the implementation, which means that the $\triangle M_{2}$ is integrated to $X_{i}$ so that a new $X_{i}$ is obtained, and the $y_{i}$ will be substituted by a supervised time series with zero-mean short memory in mean. The corresponding nonlinear estimation function is thus denoted as follows:

$$
f(\cdot)=f\left(r_{3-m}, r_{30-y}, r_{\text {bank }}, \Delta_{\text {income }}, \Delta_{\text {index }}^{+}, \Delta_{\text {index }}^{-}, \Delta M_{2}\right) .
$$

Next, a estimated output value $\left\{\hat{y}_{i}\right\}$ comes arise based on the function $f(\cdot)$, with the new $X_{i}$ as the input vector again. By the nonlinear cointegration discriminate analysis using Eviews statistic software, the $\left\{\hat{y}_{i}\right\}$ is not long-term memory. Hence, it is concluded that there really exists a nonlinear cointegration relation among the $\triangle M_{2}$ and its influencing factors, such as $r_{3-m}, r_{30-y}, r_{\text {bank }}, \Delta_{\text {income }}, \Delta^{+}{ }_{\text {index }}$, and $\Delta_{\text {index. }}^{-}$.

At the same time, the empirical results based on other nonlinear cointegration discriminate analysis methods are also carried out so that their performance can be compared. The corresponding modified $R / S$ values under the statistical value $0.4<\mathrm{p}<0.5$ is shown in Table II .

TABLE II. THE COMPARATION OF MODEL PERFORMATION BETWEEN THE SUPPORT VECTOR MACHINE AND OTHER APPROACHES

\begin{tabular}{|c|c|c|}
\hline & $\alpha=\mathbf{5 \%}$ & $\alpha=\mathbf{1 0} \%$ \\
\hline The NLS & 1.2734 & 1.2148 \\
\hline The NN & 1.2916 & 1.2478 \\
\hline The $\varepsilon$-SVR & 1.2873 & 1.2167 \\
\hline
\end{tabular}

It is not difficult to see that for the different conference degree $\alpha=5 \%$ and $\alpha=10 \%$, the modified $R / S$ values in the $\varepsilon$-SVR are all more adjacent to those in the statistical approach than that in the neural network. This means that the $\varepsilon$-SVR can seize the nonlinear relations among the $\triangle M_{2}$ and its influencing factors. This demand that the monetary policymakers should pay close attention to the changes in stock market so that the monetary policy can preferably reflect the impact from the stock prices.

And at the same time, these models above are also compared by using the other procedure for efficiency of any model defined by Nash and Sutcliffe (1970) [27], $R^{2}$, which is a criterion of determining the efficiency of a model in comparison with the average value of data. It is defined as:

$$
R^{2}=1-\frac{\sum_{i=1}^{l}\left(y_{i}-\hat{y}_{i}\right)^{2}}{\sum_{i=1}^{l}\left(y_{i}-\bar{y}\right)^{2}}
$$

where $\bar{y}$ is the average value of observed data. This criterion does not indicate the degree of interrelation (namely, correlation coefficient) between estimated and observed values, but it implies the efficiency of the model used. A value of $R^{2}$ close to 1 indicates information for linear dependence agreement between the estimated and observed values. The $R^{2}$ values of different models defined in (6) are also computed and shown in Table III.

TABLE III. THE COMPARATION OF $R^{2}$ VALUES OF DIFFERENT MODELS

\begin{tabular}{|c|c|}
\hline & $\boldsymbol{R}^{2}$ \\
\hline The NLS & 0.7054 \\
\hline The NN & 0.8191 \\
\hline The $\varepsilon$-SVR & 0.8369 \\
\hline
\end{tabular}


The $R^{2}$ value of the $\varepsilon$-SVR takes 0.8369 , being biggest among all models and having weaker advantage in the linear dependence agreement than the $\mathrm{NN}$, because the decision function represented in (2) displays the linear dependence correlation between the estimated and observed values with the kernel function working in the feature space. Obviously, the selection and option of the kernel function in (2) will become critical to the decision of the linear dependence agreement.

\section{CONCLUSIONS}

The nonlinear analysis methods are continuously emerging and the research towards their analysis mechanism of the nonlinear cointegration is deepening day by day. As a kind of effective artificial intelligence technology, the support vector machine is playing more important role in the innovation of nonlinear cointegration analysis. Compared with the neural network already used in the nonlinear cointegration analysis, the support vector machine shows more satisfactory performance in model generation and stability just demonstrated in empirical analysis, and implements the key step in the nonlinear cointegration test satisfactorily. What is to do next for the methodology will constitute a great challenge in which much importance should be attached to the selection and option of the kernel function and the self-adaptive adjustment of the model structure to the economic variables.

And as for the application to the analysis of the connection between financial markets, the next work will be concentrated on analyzing the high-order effect of input variables and capturing the uncertain volatility in the states of the economy, which will further improve the robustness of the model proposed.

\section{ACKNOWLEDGMENT}

The work is sponsored by the national natural science fund (Grant NO. 70971079) and Shandong Province Natural Science Foundation (Grant NO. ZR2011GM012, NO. ZR2012GM006).

\section{REFERENCES}

[1] Granger C W J, "Some properties of time series data and their use in econometric model specification”, Journal of Econometrics, vol. 16, pp. 121-130, 1981.

[2] Engle R, Granger C W J, "Cointegration and error correction: representation, estimation and testing”, Econometrica, vol. 55, pp. 251-276, 1987.

[3] Johansen S, "Statistical analysis of cointegration vectors", Journal of Economic Dynamics and Control, vol. 12, no. 2-3, pp. 231-254, 1988.

[4] Johansen S, "Estimation and hypothesis testing of cointegration vectors in gaussian vector autoregressive models”, Econometrica, vol. 59, no. 6, pp. 1551-1580, 1991.

[5] Dufrénot G, Mignon V, "Recent developments innonlinear cointegration with applications to macroeconomics and finance", New York: Kluwer Academic Publishers, 2002.

[6] Chang Y, Park J Y, "Index models with integrated time series", Journal of Econometrics, vol. 114, no. 1, pp. 73-106, 2003.
[7] Lieberman O, Phillips P C B, "Refined inference on long memory in realized volatility”, Econometric Reviews, vol. 27, no. 1-3, pp. 254267, 2008.

[8] Choi I, Saikkonen P, “Tests for nonlinear cointegration”, Cambridge: Econometric Theory-Cambridge University Press, 2010.

[9] Granger C W J, Hallman J, "Long memory series with attractors", Oxford Bulletin Economics and Statistics, vol. 53, no. 1, pp. 1126,1991.

[10] Chang Y, Park J Y, Phillips P C B, "Nonlinear econometric models with cointegrated and deterministically trending regressors", Econometrics Journal, vol. 4, no. 1, pp. 1-36, 2001.

[11] Zhang S Y, Xu Q F, Zhou H, "Financial time series analysis”, Beijing: Tinghua university press, 2008 [in Chinese].

[12] Escribano A, Sipols E, Aparicio F, "Nonlinear cointegration and nonlinear error correction: record counting cointegration tests", Communications in Statistics-Simulation and Computation, vol. 35, no. 4, pp. 939-956, 2006.

[13] Esteve V, Prats M A, "Threshold cointegration and nonlinear adjustmentbetween stock prices and dividends”, Applied Economics Letters, vol. 17, no. 4, pp. 405-410, 2010.

[14] Karlsen H A, Myklebust T, Tjøstheim D, "Nonparametric estimation in a nonlinear cointegration type model", Annals of Statistics, vol. 35, no. 1, pp. 252-299, 2007a.

[15] Karlsen H A, Myklebust T, Tjøstheim D, "The asymptotic properties of nonparametric estimators either for nonlinear error correction or cointegration models”, The Annals of Statistics, vol. 35, pp. 252-299, 2007b.

[16] Vapnik V, "The nature of statistical learning theory”, New York: Springer, 1995.

[17] Cristianini J S T N, Support vector machines and other kernel-based learning methods, Cambridge: Cambridge University Press, 2000.

[18] Vapnik V, Golowich S E, Smola A, "Support vector method for function approximation, regression estimation, and signal processing", In M. Mozer, M. Jordan, and T. Petsche (Eds.), Advances in Neural Information Processing Systems 9. Cambridge, MA: The MIT Press, 1997.

[19] Drucker H, Burges C J C, Kaufman L, Smola A, Vapnik V, "Support vector regression machines”, In M. Mozer, M. Jordan, and T. Petsche (Eds.), Advances in Neural Information Processing Systems 9. Cambridge, MA: The MIT Press, 1997.

[20] Shen C H, Wang X R, "Analysis of convertible bond value based on integration of support vector machine and copula function", Communications in Statistics-Simulation and Computation, vol. 40, no. 10, pp. 1563-1575, 2011.

[21] Amano T, Kato T, Taniguchi M, "Statistical estimation for CAPM with long-memory dependence”, Advances in Decision Sciences, pp. 1-12, 2012.

[22] Rigobon R, Sack B, "Measuring the reaction of monetary policy to the stock market", NBER working paper, Cambridge: National bureau of economic research, 2001.

[23] Johnson H, "Monetary theory and policy", American Economic Review, vol. 52, no. 3, pp. 335-384, 1962.

[24] Friedman M, "Money and the stock market", Journal of Political Economy, vol. 96, no. 2, pp. 221-245,1988.

[25] Li G, "A new challenge from the development of the capital market to the monetary policy”, Beijing: Graduate School Chinese Academy of Social Sciences, 2001 [in Chinese].

[26] Cao L J, Chua K S, Gu Q M, Lee H P, “Saliency analysis of support vector machines for gene selection in tissue classification”, Neural Computing and Applications, vol. 11, no. 3-4, pp. 244-249, 2003.

[27] Nash J E, Sutcliffe J V, "River flow forecasting through conceptual models, Part I: A discussion of principles”, Journal of Hydrology, vol. 10, no. 3, pp. 282-290, 1970. 